\title{
Genotypic and Phenotypic Characterization of Clinical Escherichia coli Sequence Type 405 Carrying IncN2 Plasmid Harboring bla NDM-1
}

\author{
Yingying Hao, Chunhong Shao, Xu Geng, Yuanyuan Bai, Yan Jin* and Zhiming Lu* \\ Department of Clinical Laboratory, Shandong Provincial Hospital Affiliated to Shandong University, Jinan, China
}

OPEN ACCESS

Edited by:

Benjamin Andrew Evans, University of East Anglia,

United Kingdom

Reviewed by:

Abdelaziz Touati,

University of Béjaïa, Algeria

Yang Liu,

The First Affiliated Hospital

of Nanchang University, China

${ }^{*}$ Correspondence:

Yan Jin

sdslyyjy@163.com

Zhiming Lu

luzhiming@sdu.edu.cn

Specialty section:

This article was submitted to Antimicrobials, Resistance

and Chemotherapy,

a section of the journa

Frontiers in Microbiology

Received: 07 January 2019 Accepted: 27 March 2019

Published: 12 April 2019

Citation:

Hao Y, Shao C, Geng X, Bai Y, Jin $Y$ and Lu Z (2019) Genotypic and Phenotypic Characterization of Clinical Escherichia coli Sequence Type 405 Carrying IncN2 Plasmid Harboring bla ${ }_{N D M-1}$.

Front. Microbiol. 10:788. doi: 10.3389/fmicb.2019.00788

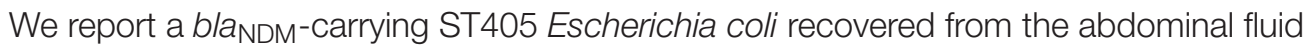
of a patient in Shandong, China. This strain belonged to the high-risk phylogenetic group $\mathrm{D}$ and carried the virulence genes, papG II, papG III, papC, and iroN. In addition to bla $\mathrm{NDM}-1_{1}$, this isolate carried the quinolone resistance gene $a c c\left(6^{\prime}\right)-I b$ and extendedspectrum $\beta$-lactamase (ESBL) genes bla $\mathrm{CTX}-\mathrm{M}-15$ and bla $\mathrm{CTX}-\mathrm{M}-14$. bla $\mathrm{NDM}_{\mathrm{N}-1}$ was located on a $41 \mathrm{~Kb}$ IncN2 self-transmissible plasmid. The IncN2 plasmid named as pJN24NDM1 was fully sequenced and analyzed. Genome comparative analysis showed that IncN2 plasmids harboring carbapenem-resistance genes possessed conserved backbones and variable accessory regions. Phylogenetic analysis of $87 \mathrm{IncN}$ plasmids based on orthologous genes indicated that 9 IncN2 plasmids fell into one phylogenetic clade, while 4 IncN3 plasmids were in two phylogenetic clades of the 74 IncN1 plasmids. The presence of IncN2 plasmids harboring bla NDM in the high-risk clone ST405 E. coli raises serious concerns for its potential of dissemination.

Keywords: bla $_{\mathrm{NDM}-1}, \mathrm{ST} 405$, Escherichia coli, IncN2, China, carbapenem-resistant Enterobacteriaceae

\section{INTRODUCTION}

New Delhi metallo-beta lactamase (NDM)-producing Enterobacteriaceae have emerged as a critical public-health problem worldwide (Ishii et al., 2017; Livorsi et al., 2018). Recently, a study from the French National Reference Center showed that $21 \%$ of 140 carbapenem-resistant isolates produced NDM carbapenemase (Gauthier et al., 2018). Nationwide surveillance of clinical carbapenemresistant Enterobacteriaceae in China showed that NDM production was predominantly associated with carbapenem resistance in Escherichia coli (Zhang et al., 2017; Li et al., 2018; Wang et al., 2018). In other countries such as India, Pakistan, and the Balkan countries, infections caused by NDM-producer has created large medical and economic burdens (Otter et al., 2017). Notably, bla $a_{\mathrm{NDM}}$-carrying strains have also been isolated from pets, food animals, and environmental samples such as sewage water and urban rivers (Hao et al., 2018). The presence of NDM-producer in the community creates an additional risk of dissemination by travelers (Zhong et al., 2017). Targeted resistance surveillance and interventions are needed to control the expanding global distribution of $b l a_{\mathrm{NDM}}$-carrying strains. 
Extraintestinal pathogenic E. coli (ExPEC) is a leading cause of severe infections and has become increasingly resistant to broader classes of antimicrobial agents (Peirano et al., 2013). NDM-producing ST405, phylogenetic group D E. coli is an emerging ExPEC pathogen with sporadic reports from Canada, Denmark, Spain, the United Kingdom, Pakistan, Italy, India, and China (Jakobsen et al., 2014; Peirano et al., 2014; Toleman et al., 2015; Ranjan et al., 2016; Barrado et al., 2018; Qamar et al., 2018; Zhang et al., 2018). bla $a_{\mathrm{NDM}}$-carrying IncB/O, IncFII, IncFIA, and IncFIb type plasmids have been identified in ST405 E. coli strains.

In this study, we report a ST405 E. coli isolate producing $b l a_{\mathrm{NDM}-1}$ harbored by an IncN2 plasmid recovered from a patient with intra-abdominal infection in our hospital. This is the first report of the bla $a_{\mathrm{NDM}}$-harboring IncN2 plasmid carried by ST405 E. coli. There are only 8 fully sequenced IncN2 plasmids harboring carbapenemase in the National Center for Biotechnology Information (NCBI) database. The molecular characteristics of the IncN2 plasmid harboring bla $a_{\mathrm{NDM}}$ were determined by genomic comparison and phylogenetic analyses.

\section{MATERIALS AND METHODS}

\section{Clinical Case, Clinical Strain, and Susceptibility Assays}

The carbapenem-resistant E. coli strain JN24 was isolated from a 58-year old man with abdominal infection who had been admitted to a teaching hospital in Shandong, China in 2015. Pancreaticoduodenectomy was conducted for the patient to treat distal bile duct carcinoma. Abdominal infection and a persistent high temperature of $39.5^{\circ} \mathrm{C}$ was observed at 1 week after surgery. The patient was administered intravenously with imipenem and vancomycin, but he failed to respond to the treatment. His condition deteriorated, and he quit therapy in the next week because of the financial burden of the treatment. E. coli JN24 was recovered from the abdominal fluid of this patient. Although blood culture was not performed, bacteremia was highly suspected. The patient had no history of travel. His medication history and hospital course were obtained from the hospital information system. The case history collection and report were approved by the Ethics Committee of Shandong Provincial Hospital. Species identification of strain JN24 was conducted with the VITEK2 automated system (BioMérieux, Marcy-l'Étoile, France) in the clinical microbiology laboratory of Shandong Provincial Hospital. The carbapenem inactivation method (CIM) and EDTA-modified CIM method were performed for phenotypic detection of carbapenemases.

Minimum inhibitory concentration (MIC) testing was conducted using E-test Strips for aztreonam, cefepime, cefotaxime, cefoxitin, ceftazidime, ertapenem, imipenem, meropenem, piperacillin-tazobactam, trimethoprim-sulfamethoxazole, ciprofloxacin, gentamicin, amikacin, polymyxin B, fosfomycin, and tigecycline. The MIC results were interpreted using Clinical Laboratory Standards Institute criteria (CLSI, 2017), except for polymyxin B and fosfomycin, which were interpreted using the EUCAST criteria (EUCAST, 2017).

\section{Molecular Typing and Phylogenetic Group Genotyping}

Multilocus sequence typing (MLST) of the isolate was conducted using a standard method according to the guidelines on the E. coli MLST website (Bitar et al., 2017). The phylogenetic group of JN24 was determined by a multiplex PCR-based method using the primers chuA, yjaA, and TspE4.C2 (Wirth et al., 2006). Multiplex PCR assays were conducted to detect virulence-associated genes as described previously (Wang L. H. et al., 2016).

\section{Molecular Detection of Resistance Genes}

Antimicrobial resistance genes were detected by PCR as described previously (Zhu et al., 2016). These antimicrobial resistance genes included carbapenemase-encoding genes, extended-spectrum $\beta$-lactamase genes, plasmid-mediated pAmpC $\beta$-lactamase, $16 \mathrm{~S}$ rRNA methylase genes, plasmid-mediated fosfomycin resistance genes, plasmid-mediated quinolone resistance genes, and polymyxin B resistance genes $(\mathrm{mcr}-1)$, which have been described previously (Du et al., 2016; Zhu et al., 2016).

\section{bla $a_{\mathrm{NDM}}$-Carrying Plasmid Analysis and Sequencing}

Plasmid conjugation experiments were performed as described previously using E. coli J53Azi ${ }^{\mathrm{R}}$ as the recipient and strain JN24 as the donor. Transconjugants were screened on Mueller-Hinton agar plates with sodium azide $(100 \mu \mathrm{g} / \mathrm{mL})$ and ceftazidime $(6 \mu \mathrm{g} / \mathrm{mL})$. The transconjugant J24 and recipient J53AziR were subjected to antimicrobial susceptibility testing using a method similar to that used for the clinical strain.

S1 pulsed field gel electrophoresis (S1-PFGE) was conducted to evaluate the size and amounts of plasmids carried by the clinical strain JN24 and transconjugant J24. Southern blotting was performed to determine the location of $b l a_{\mathrm{NDM}-1}$. The genomic DNA of the clinical strain and transconjugant embedded in the gel plugs was digested with QuickCut S1 nuclease (Takara, Shiga, Japan) for $1 \mathrm{~h}$, and then separated by PFGE for $17 \mathrm{~h}$ with the pulse time switched from 2.16 to 63.8 s. Salmonella strain H9812 was digested with QuickCut XbaI (Takara, Shiga, Japan) and used as the reference marker (Liu et al., 2018). Plasmid DNA was transferred to a nylon membrane and analyzed by Southern blotting using specific bla $a_{\mathrm{NDM}}$ probes labeled with digoxigenin (Roche, Basel, Switzerland) (Liu et al., 2018).

We tried to eliminate pJN24NDM1 from the clinical strain JN24 to evaluate the antibiotic resistance profile of JN24 without the NDM-plasmid. A culture of the clinical isolate JN24 was grown overnight at $37^{\circ} \mathrm{C}$ on a Luria-Bertani agar plate, and then transferred to a fresh Luria-Bertani agar plate. After repeated passage for 10 days, 10 colonies were randomly selected and screened for bla $a_{\mathrm{NDM}}$ by PCR (Zhu et al., 2016).

Genomic DNA of the plasmid carrying $b l a_{\mathrm{NDM}}$ was prepared using the Wizard ${ }^{\circledR}$ Genomic DNA Purification Kit (Promega, Madison, WI, United States) and sequenced with $150 \times$ coverage using an Illumina HiSeq X Ten (San Diego, CA, United States) at MajorBio Co. (Shanghai, China). Reads were trimmed and 
TABLE 1 | Antimicrobial resistance profile of Escherichia coli JN24 and the transconjugant J24.

\begin{tabular}{|c|c|c|c|c|c|c|c|c|c|c|c|c|c|c|c|c|c|}
\hline \multirow[b]{2}{*}{ isolates } & \multicolumn{17}{|c|}{ MIC $(\mu \mathrm{g} / \mathrm{mL})$} \\
\hline & CZO & CRO & CAZ & FOX & FEP & ATM & TZP & MEM & ETP & IMP & SXT & AK & $\mathrm{CN}$ & CIP & LEV & FOS & TGC \\
\hline J53Azi ${ }^{R}$ & $<=0.016$ & $<=0.016$ & $<=0.016$ & $<=0.016$ & $<=0.016$ & $<=0.016$ & $<=0.016$ & $<=0.02$ & $<=0.02$ & $<=0.02$ & $<=0.02$ & $<=0.016$ & $<=0.016$ & $<=0.02$ & $<=0.02$ & 2 & 0.38 \\
\hline $\mathrm{J} 24$ & $>=256$ & $>=256$ & $>=256$ & $>=256$ & 16 & 0.64 & $>=256$ & 8 & 12 & 16 & 0.32 & 1 & 0.5 & $<=0.02$ & $<=0.02$ & 2 & 0.38 \\
\hline JN24 & $>=256$ & $>=256$ & $>=256$ & $>=256$ & $>=256$ & $>=256$ & $>=256$ & $>=32$ & $>=32$ & $>=32$ & $>=32$ & $>=256$ & $>=256$ & $>=32$ & $>=32$ & 2 & 0.38 \\
\hline
\end{tabular}

MIC, minimal inhibitory concentrations; CZO, cefazolin; CRO, cefatriaxone; CAZ, ceftazidime; FOX, cefoxitin; FEP, cefepime; ATM, aztreonam; TZP, piperacillin/tazobactam; MEM, meropenem; ETP, ertapenem; IPM, imipenem; STX, trimethoprim/sulfamethoxazole; AK, amikacin; CN, gentamicin; CIP, ciprfloxacin; LEV, Levofloxacin; FOS, Fosfomycin; TGC, tigecycline.

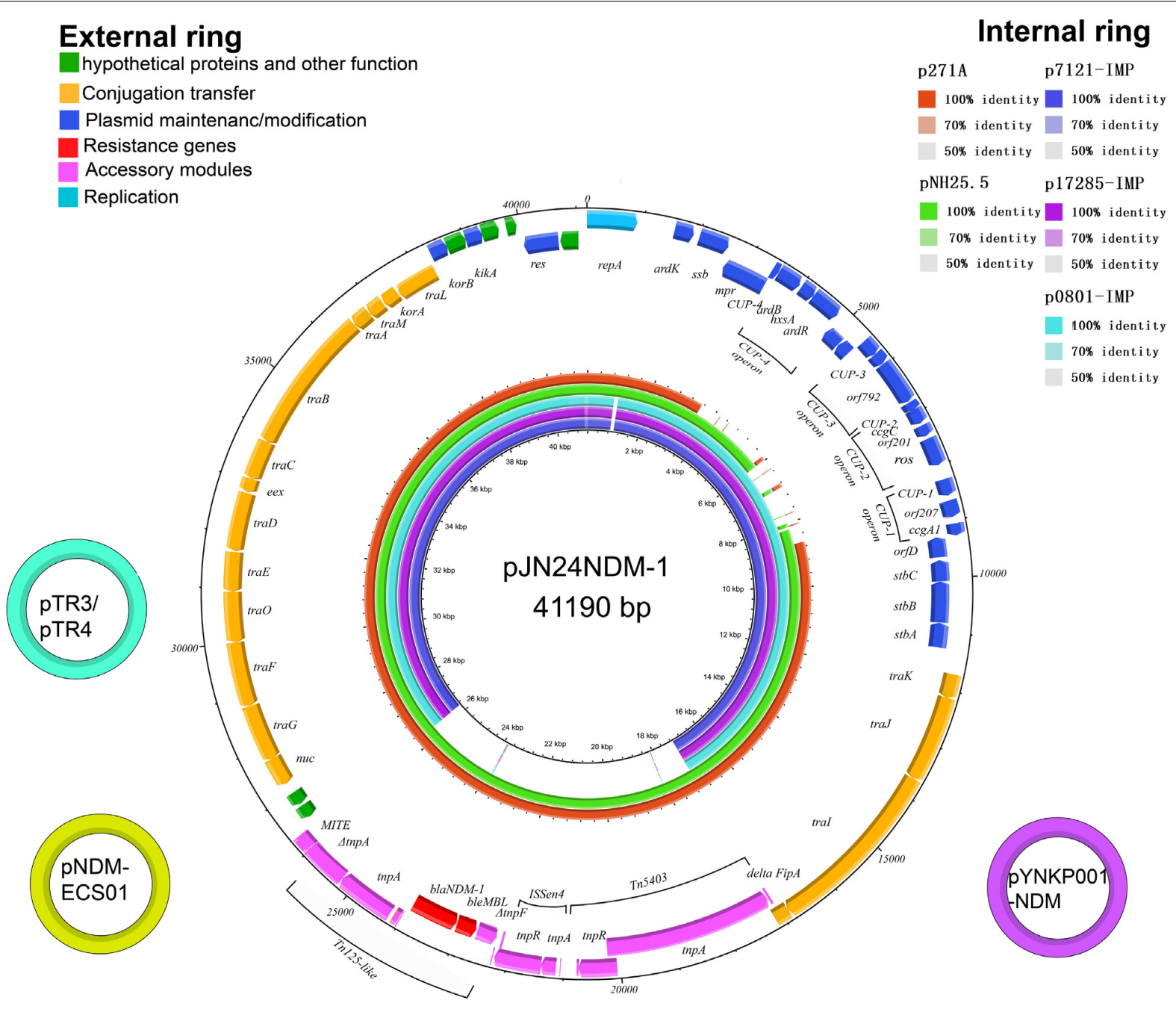

FIGURE 1 | The external ring is the schematic representation of plasmid pJN24NDM1 (GenBank accession no. MK368725). The three small external rings display the IncN2 type plasmids harboring bla $\mathrm{NDM}-1_{1}$ with identity $>99 \%$. The genes with different functional annotations were labeled with different colors. The internal five rings showed the comparative analysis of blaNDM-harboring IncN2 plasmids with pJN24NDM1, including p7121-IMP (purple), p17285-IMP (violet), p0801-IMP (sky blue), p271A (orange), and pNH25.5 (green) (constructed by BRIG).

assembled using SOAPdenovo2. PCR and Sanger sequencing were conducted at Sangon Biotech (Shanghai, China) to close the gaps. The plasmid sequence was screened for resistance genes using the Comprehensive Antibiotic Resistance Database ${ }^{1}$

${ }^{1}$ https://card.mcmaster.ca/ and the plasmid type was established using PlasmidFinder at the Center for Genomics and Epidemiology ${ }^{2}$. Coding sequence prediction of the plasmid was performed with Rapid Annotations using Subsystems Technology and annotation of the predicted

\footnotetext{
${ }^{2}$ https://cge.cbs.dtu.dk/services/
} 
open reading frames was conducted by blast against the nonredundant protein database. Mobile elements were detected using ISFinder and the Tn Number Registry.

\section{Genome Comparisons}

For comparative analysis, 86 plasmids harboring carbapenemases with IncN1, IncN2, or IncN3 type replicons were downloaded from the NCBI. The presence of carbapenemases was screened by blasting against the Comprehensive Antibiotics Resistance database. Comparative analysis of the genetic contexts among IncN plasmids was conducted using a Large-Scale Blast Score Ratio (LS-BSR) pipeline (Hazen et al., 2018) and displayed in a pheatmap. Eight fully sequenced IncN2 plasmids harboring carbapenemases were compared to pJN24NDM1 by BLAST Ring Image Generator (Liu et al., 2018). More detailed genome alignment was conducted by local BLAST and visualized with Easyfig (Zhu et al., 2016).

\section{Phylogenetic Analysis of IncN Plasmids Harboring Carbapenemases}

Among the 87 plasmids, homologs were identified using the OrthoFinder and a set of 56 genes representing the core genome was summed. Orthologous genes were aligned by Multiple Alignment using Fast Fourier Transform, and the maximumlikelihood phylogenetic tree was inferred using RAxML with a 1000-bootstrap test (Long et al., 2019).

\section{RESULTS AND DISCUSSION}

\section{Strain Features}

The antibiotic MICs of E. coli strain JN24 are listed in Table 1. The strain was resistant to quinolones, trimethoprimsulfamethoxazole, piperacillin-tazobactam, carbapenems, aztreonam, and cephalosporins but remained susceptible to polymyxin B, aminoglycosides, fosfomycin, and tigecycline. The isolate was established as a metallo-beta-lactamase (MBL)producer by CIM and EDTA-modified CIM. In addition to $b l a_{\mathrm{NDM}-1}$, the strain was found to carry bla $a_{\mathrm{CTX}-\mathrm{M}-15}$, bla $a_{\mathrm{CTX}-\mathrm{M}-14}$, and the plasmid-mediated quinolone resistance gene $a c c\left(6^{\prime}\right)-I b$. Therefore, this strain was highly resistant to $\beta$-lactam drugs and showed multidrug resistance. Plasmidmediated $\mathrm{pAmpC} \beta$-lactamase, fosfomycin resistance genes, and the colistin $\mathrm{B}$ resistance gene were not detected.

The clinical E. coli strain JN24 was assigned to the highrisk clone ST405 and phylogenetic group D. This strain carried multiple virulence genes, including kpsMT III type cps, papG II, papG III, papC, and iroN, which enhanced its adhesion, colonization, and Fe availability. According to a multicenter study of E. coli bloodstream infections in Shanghai, ST131 belonging to phylogroup B2 and ST405 belonging to phylogroup D contributed briefly to the dissemination of EC-BSI in Shanghai (Wang S. et al., 2016). Notably, 3 of the 6 carbapenem-resistant isolates were D-ST405 E. coli. Other studies also reported that ST405 E. coli isolates harboring CTX-M may be related to NDM $\beta$-lactamase production (Wang S. et al., 2016). Additional studies focused on the reliable molecular surveillance of high-risk clones with MDR are needed to prevent their further dissemination.

\section{Characterization of bla NDM- $^{-}$ Harboring Plasmid}

The plasmid pJN24NDM1 from strain JN24 harboring the New Delhi metallo-beta-lactamase was successfully transferred into E. coli $\mathrm{J} 53 \mathrm{Azi}^{\mathrm{R}}$ by conjugation. The presence of $b l a_{\mathrm{NDM}-1}$ and absence of bla $a_{\mathrm{CTX}-\mathrm{M}-15}, b l a_{\mathrm{CTX}-\mathrm{M}-14}$, and $\operatorname{acc}\left(6^{\prime}\right)-\mathrm{Ib}$ in the transconjugant J24 was confirmed by PCR. The transconjugant was resistant to carbapenems and cephalosporin but was susceptible to quinolones, aminoglycosides, and aztreonam.

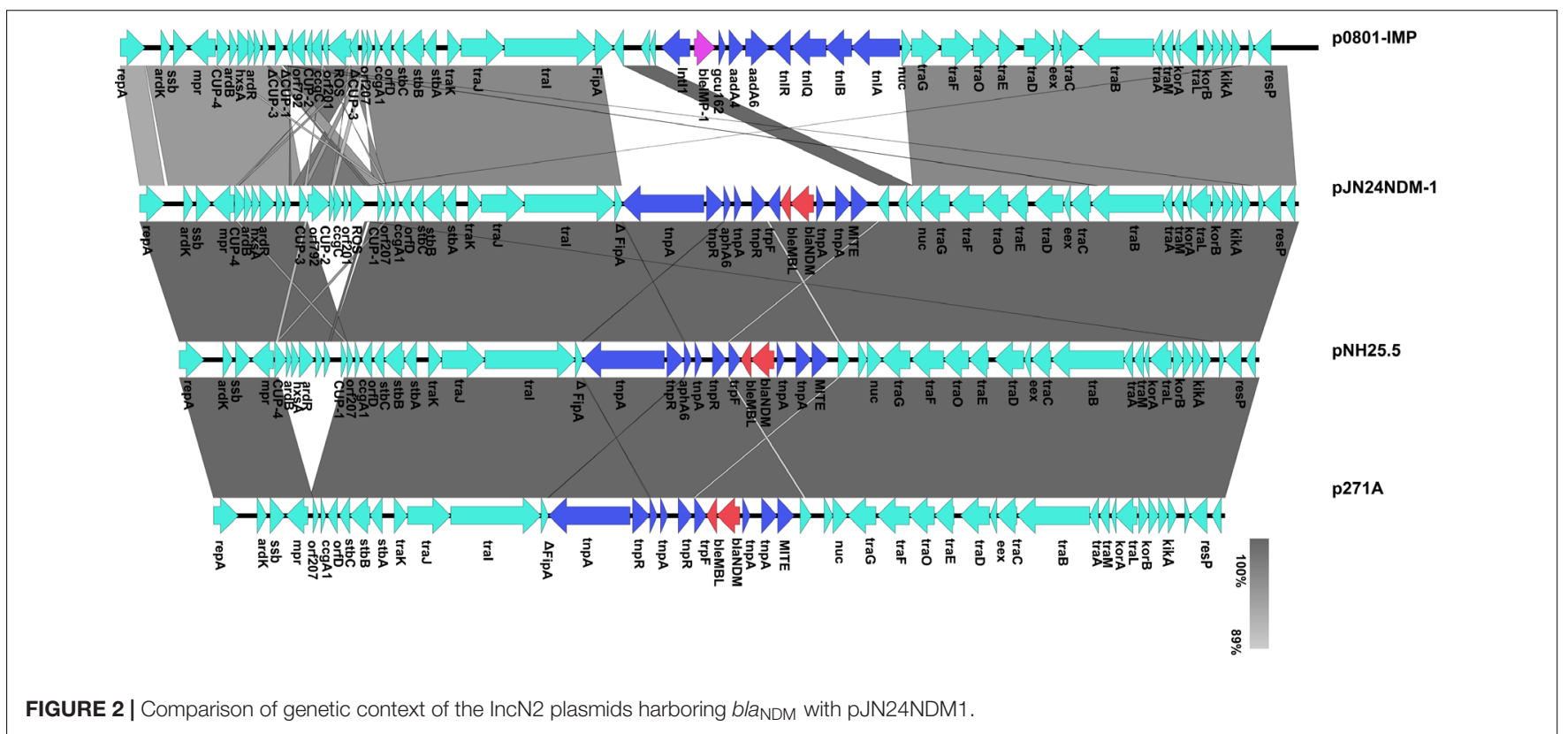




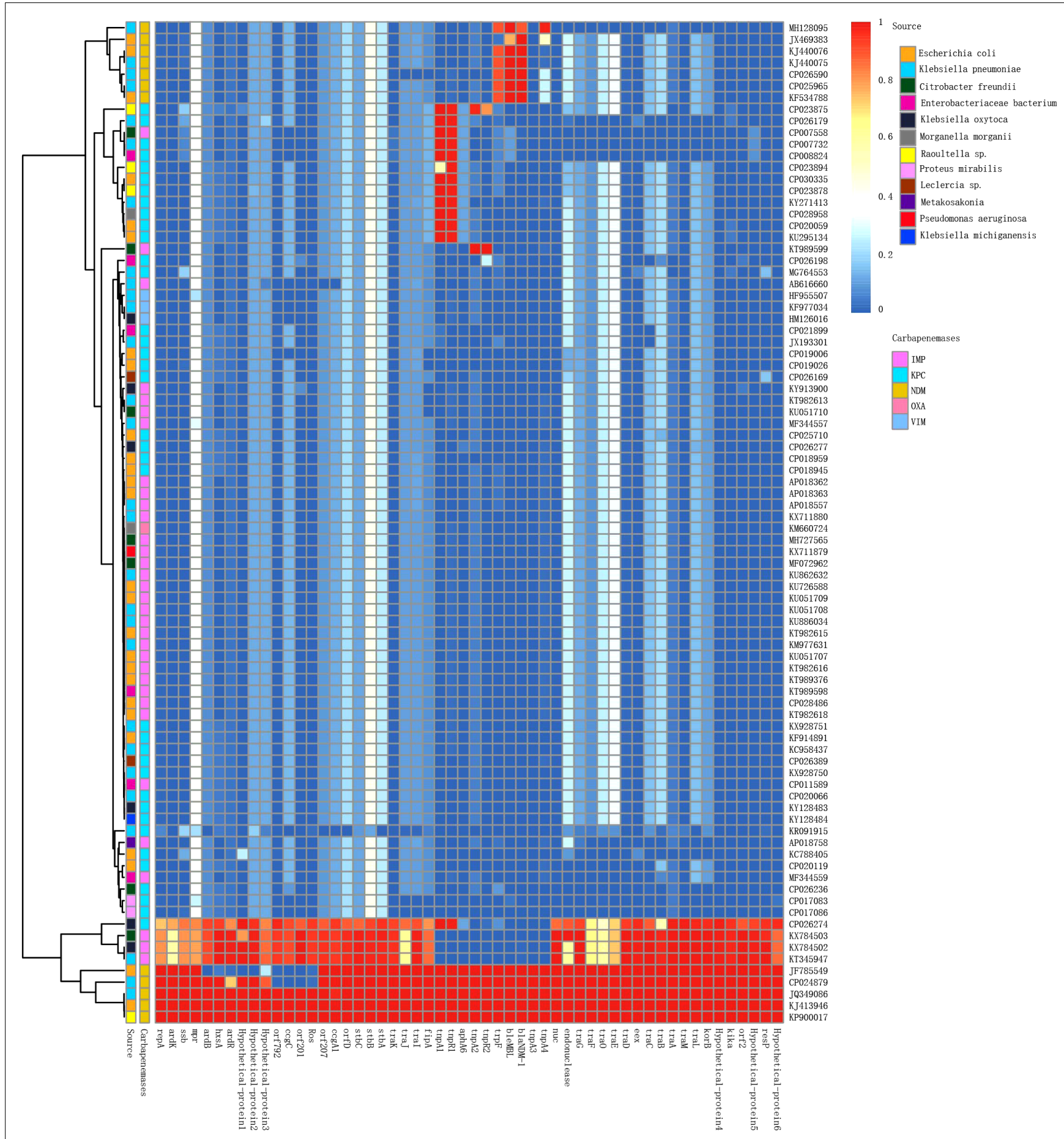

FIGURE 3 | Detection of plasmid pJN24NDM1 genes in the IncN plasmids harboring carbapenemases analyzed in this study. The BLAST score ratio (BSR) values in the heatmap represented the existence (red) or non-existence (blue) of gene of the plasmid pJN24NDM1 in each of the plasmids. Rows represented each of the CDS of pJN24NDM1, while the column represented a different palsmid. The source and carbapenemase of each plasmids were marked by squares at the top of the heat map. The detailed information of the plasmids was listed in the Supplementary Table S1.

S1-PFGE and Southern blot analysis revealed that the clinical strain JN24 carried two plasmids. bla $a_{\mathrm{NDM}}$ was located on the plasmid and showed a size of approximately $41 \mathrm{~kb}$ (Supplementary Figure S1). We failed to eliminate the plasmid harboring $b l a_{\mathrm{NDM}}$ from the clinical isolate JN24, indicating that this plasmid was stable in isolate JN24.

The complete plasmid sequence was determined to better characterize the plasmid pJN24NDM1 harboring bla $a_{\mathrm{NDM}-1}$. 


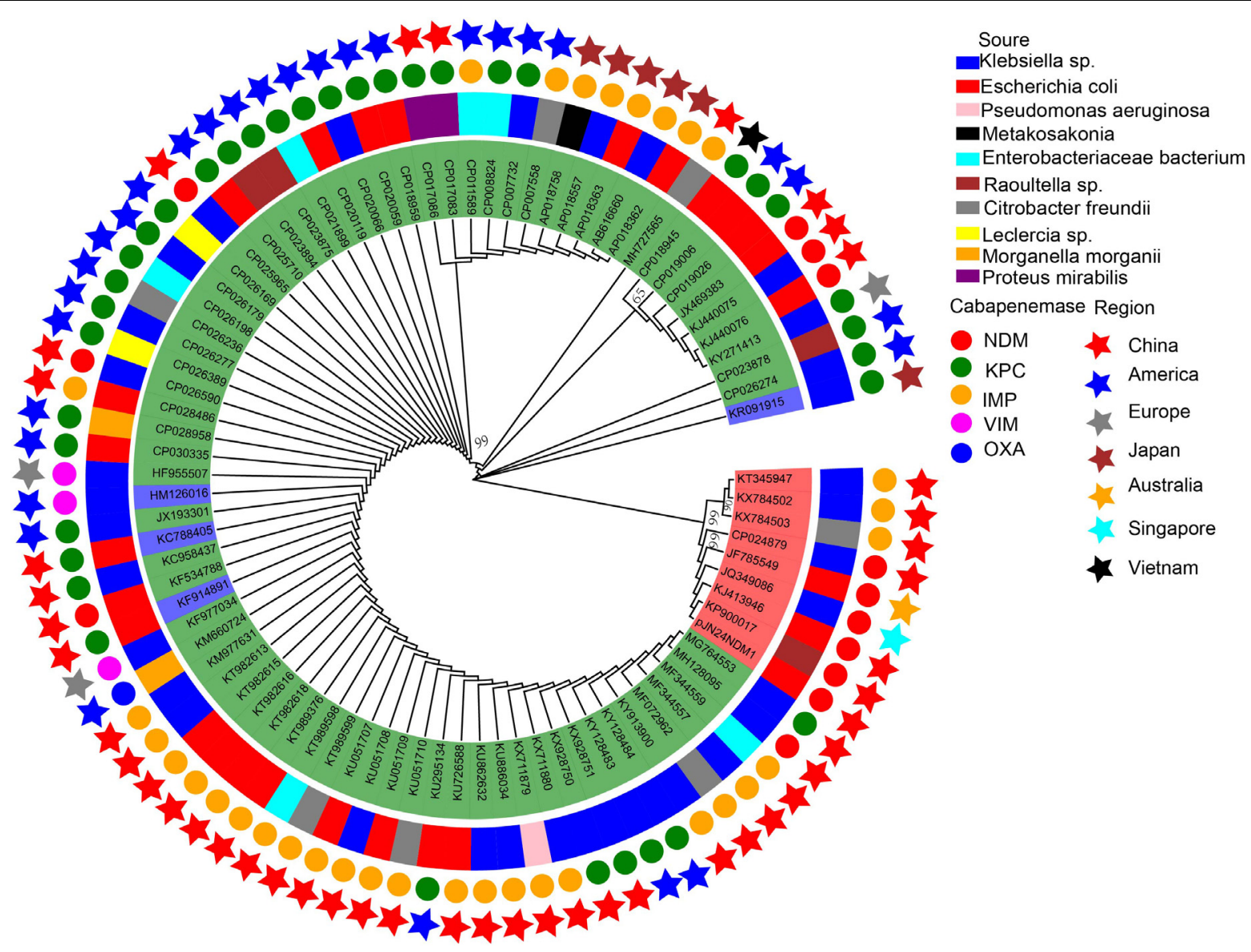

FIGURE 4 | Phylogenetic analysis of IncN plasmids harboring carbapenemases.

pJN24NDM1 is a 41,190-base pair (bp) plasmid that belongs to the IncN2 incompatibility group according to replication module analysis. In pJN24NDM1, the genetic environment of $b l a_{\mathrm{NDM}}$ in pJN24NDM1 was MITE- $\operatorname{tnp} A-I S E c 33-b l a_{\mathrm{NDM}^{-}}$ bleMBL- $\Delta$ tnpF-ISSen4- $\triangle a p h A 6-T n 5403$ and that in IncX3 was

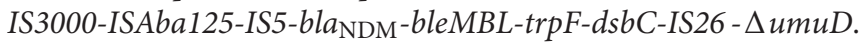
$b l a_{\mathrm{NDM}-1}$ was embedded by a miniature inverted repeat element (MITE) and ISAba125 was interrupted by ISEc33 in the upstream sequence, followed by ble-ISSen4-Tn5403 in the downstream region. The insertion of Tn125-like elements by MITE in IncN2 plasmids indicated mobilization of $b l a_{\mathrm{NDM}-1}$. The Tn125-like element in pJN24NDM1 lacked the fragments of $d s b C, \operatorname{cutA}$, groES, groEL, and ISCR27, which are present in the classical Tn125 element (Jiang et al., 2017; Hao et al., 2018).

\section{Comparative Analysis of IncN2 Plasmids Harboring Carbapenemases}

Eight fully sequenced IncN2 plasmids harboring carbapenemases obtained from the NCBI database were compared to pJN24NDM1, including pTR3 (GenBank accession no. JQ349086), pNDM-ECS01 (GenBank accession no. KJ413946), pYNKP01-NDM (GenBank accession no. KP900017), p271A
(GenBank accession no. JF785549), pNH25.5 (GenBank accession no. NZ_CP024879), p7121-IMP (GenBank accession no. KX784502), p17285-IMP (GenBank accession no. KX784503), and p0801-IMP (GenBank accession no. KT345947) (Figure 1; Poirel et al., 2011; Chen et al., 2012; Netikul et al., 2014; Jiang et al., 2017).

Sequence alignment by BRIG showed that pJN24NDM1 was identical to the previously reported plasmid pNDM-ECS01 from ST131 E. coli isolated from Thailand and China and the plasmid pYNKP01-NDM from Raoultella ornithinolytica isolated from Mainland China (Netikul et al., 2014; Sun et al., 2015). Additionally, the pJN24NDM1 plasmid showed 99\% nucleotide identity with plasmid pTR3 of Klebsiella pneumoniae isolated from Singapore (Chen et al., 2012). These three locations are geographically contiguous, but a history of travel was denied by our patient. Thus, the IncN2 plasmids may have exit in these locations naturally or are highly transmissible. pJN24NDM1 was similar to the plasmids p17285-IMP, p7121-IMP, and p0801-IMP isolated from China, but were in different regions and the NDM cassette was replaced with the IMP cassette and a putative iteron of repA (Figure 1).

To determine the detailed structural differences between the plasmids pJN24NDM1, p271A, pNH25.5, pYNKP01-NDM, and 
p0801-IMP, additional linear comparative genomics analysis of these four plasmids was performed by BLAST. The plasmids showed similar genomic contents and varied mainly at the conserved upstream repeat (CUP) region and accessory region. Compared to pJN24NDM1, p271A lacked a 5,243-bp CUPcontrolled regulon region, possibly because of recombination between CUP sequences. Deletion of CUP-2 and CUP-3 was also observed in pNH25.5. In p0801-IMP, CUP-3 and CUP-1 were disrupted and recombined, leading to inversion of orf 792 to the ROS region. Compared to pJN24NDM1, the accessory region harboring a resistance gene was replaced with a class 1 integron In1223 in p0801-IMP (19) (Figure 2).

\section{Genomic and Phylogenetic Analysis}

The 86 IncN plasmids carrying carbapenemases downloaded from the NCBI database were sub-grouped into three types according to their replication genes: IncN1 $(n=74)$, IncN2 $(n=8)$, and IncN3 $(n=4)$ (Supplementary Table S1; Kim et al., 2016). Multiple carbapenemases were detected in these plasmids including IMP, VIM, NDM, KPC, and OXA.

IncN plasmids have been reported globally but are mainly prevalent in China and the United States. Of the 38 IncN1 plasmids reported in China (including the mainland and Taiwan, China), the major carbapenemase type was bla $\operatorname{IMP}_{\mathrm{IM}}(63.9 \% ; 23 / 36)$, followed by bla $a_{\mathrm{NDM}}(19.4 \% ; 7 / 36)$, and bla $a_{\mathrm{KPC}}(19.4 \% ; 7 / 36)$. Of the 25 IncN1 plasmids reported in the United States, the major carbapenemase type was bla $\mathrm{KPC}$, which accounts for $88 \%$ $(22 / 25)$ of IncN1 plasmids. Seven of the eight IncN2 plasmids were reported in Asia and are mainly prevalent in China (6/8). The plasmid p271A isolated from a patient in Australia was transferred from Bangladesh in South Asia (Figure 3).

IncN plasmids were detected in 16 types of bacteria, with E. coli and $K$. pneumoniae as the most frequent reservoirs. Notably, P378-IMP harboring bla $a_{\mathrm{NDM}}$ was recovered from Pseudomonas aeruginosa, which is an important nonfermentative bacterium causing hospital-acquired infections. The recombinogenic capability may endow broad host plasmids to spread multidrug resistance to other bacteria species.

To compare the genetic contents of three subtype IncN plasmids, the LS-BSR pipeline was used. pNDM-ECS01 was used as the reference genome for LS-BSR, considering that the $5243 \mathrm{bp}$ within the CUP controlling the regulon in p271A (first sequenced IncN2 plasmid) was absent. The collection of IncN2 plasmids showed a backbone arrangement similar to the IncN1 and IncN3 collection, while nucleotide sequence homology showed limited similarity (Figure 3). According to

\section{REFERENCES}

Barrado, L., Perez-Vazquez, M., Del Pozo, J. L., Martin-Salas, C., Leiva, J., Mazon, A., et al. (2018). Clonal transmission of NDM-5-producing Escherichia coli belonging to high-risk sequence type ST405. Int. J. Antimicrob. Agents 52, 123-124. doi: 10.1016/j.ijantimicag.2018.05.018

Bitar, I., Piazza, A., Gaiarsa, S., Villa, L., Pedroni, P., Oliva, E., et al. (2017). ST405 NDM-5 producing Escherichia coli in Northern Italy: the first two clinical cases. Clin. Microbiol. Infect. 23, 489-490. doi: 10.1016/j.cmi.2017.01.020 maximum-likelihood phylogenetic analysis, the IncN2 plasmids were in one phylogenetic clade, while 4 IncN3 plasmids were in 2 phylogenetic clades of 74 IncN1 plasmids (Figure 4).

\section{CONCLUSION}

In this study, a self-transmissible IncN2 plasmid bearing $b l a_{\mathrm{NDM}}$ was detected in ST405 E. coli, which is a globally disseminated lineage. We highlight the dissemination potential of IncN2 plasmids harboring $b l a_{\mathrm{NDM}}$. The presence of $b l a_{\mathrm{NDM}}$ mediated by plasmids in the high-risk clone ST405 E. coli linked with multiple virulence genes and resistance genes is increasing, which may create health concerns.

\section{AUTHOR CONTRIBUTIONS}

YJ and ZL designed the experiments. YH analyzed the data and wrote the manuscript. CS and XG prepared the table and figures. YB carried out the bacteria identification.

\section{FUNDING}

The study was supported by grants from Focus on Research and Development Plan in Shandong Province (2016GSF201078) and Jinan Science and Technology Development Plan (201805021).

\section{ACKNOWLEDGMENTS}

We would like to thank Professor Chunxiao Wu for his technical support and continuous encouragement. We would also like to thank Editage (www.editage.com) for English language editing.

\section{SUPPLEMENTARY MATERIAL}

The Supplementary Material for this article can be found online at: https://www.frontiersin.org/articles/10.3389/fmicb. 2019.00788/full\#supplementary-material

FIGURE S1 | S1-PFGE and Southern Blot patterns of clinical strain JN24 (A) and transconjugant J24 (B) M, marker; Salmonella enterica serotype H9812.

TABLE S1 | The plasmids analyzed in the large-scale BLAST score ratio analysis and Phylogenetic Analysis.

Chen, Y. T., Lin, A. C., Siu, L. K., and Koh, T. H. (2012). Sequence of closely related plasmids encoding bla (NDM-1) in two unrelated Klebsiella pneumoniae isolates in Singapore. PLoS One 7:e48737. doi: 10.1371/journal.pone.0048737

CLSI (2017). Performance Standards for Antimicrobial Susceptibility Testing; 24th Informational Supplement. Wayne, PA: Clinical and Laboratory Standards Institute (CLSI), M100-M127.

Du, H., Chen, L., Tang, Y.-W., and Kreiswirth, B. N. (2016). Emergence of the mcr1 colistin resistance gene in carbapenem-resistant Enterobacteriaceae. Lancet Infect. Dis. 16, 287-288. doi: 10.1016/S1473-3099(16)00056-6 
EUCAST (2017). European Committee on Antimicrobial Susceptibility Testing. Available at: http://www.eucast.org/clinical_breakpoints/ (accessed November 05, 2017).

Gauthier, L., Dortet, L., Cotellon, G., Creton, E., Cuzon, G., Ponties, V., et al. (2018). Diversity of carbapenemase-producing Escherichia coli isolates in france in 2012-2013. Antimicrob. Agents Chemother. 62:e266-18. doi: 10.1128/AAC. 00266- 18

Hao, Y., Shao, C., Bai, Y., and Jin, Y. (2018). Genotypic and phenotypic characterization of IncX3 plasmid carrying bla NDM-7 in Escherichia coli sequence Type 167 isolated from a patient with urinary tract infection. Front. Microbiol. 9:2468. doi: 10.3389/fmicb.2018.02468

Hazen, T. H., Mettus, R., McElheny, C. L., Bowler, S. L., Nagaraj, S., Doi, Y., et al. (2018). Diversity among blaKPC-containing plasmids in Escherichia coli and other bacterial species isolated from the same patients. Sci. Rep. 8:10291. doi: 10.1038/s41598-018-28085-7

Ishii, Y., Aoki, K., Tateda, K., and Kiyota, H. (2017). Multicenter collaboration study on the beta-lactam resistant Enterobacteriaceae in Japan - The 65th anniversary public interest purpose project of the Japanese Society of Chemotherapy. J. Infect. Chemother. 23, 583-586. doi: 10.1016/j.jiac.2017. 06.009

Jakobsen, L., Hammerum, A. M., Hansen, F., and Fuglsang-Damgaard, D. (2014). An ST405 NDM-4-producing Escherichia coli isolated from a danish patient previously hospitalized in Vietnam. J. Antimicrob. Chemother. 69, 559-560. doi: $10.1093 /$ jac/dkt356

Jiang, X., Yin, Z., Yin, X., Fang, H., Sun, Q., Tong, Y., et al. (2017). Sequencing of blaIMP-carrying IncN2 plasmids, and comparative genomics of IncN2 plasmids harboring class 1 integrons. Front. Cell Infect. Microbiol. 7:102. doi: 10.3389/ fcimb.2017.00102

Kim, S. Y., Rhee, J. Y., and Ko, K. S. (2016). Complete sequence of blaKPC2-harboring plasmid with a mosaic of IncN1- and IncN3-type plasmids in a Klebsiella pneumoniae isolate from South Korea. Antimicrob. Agents Chemother. 60, 1167-1169. doi: 10.1128/AAC.02532-15

Li, X., Fu, Y., Shen, M., Huang, D., Du, X., Hu, Q., et al. (2018). Dissemination of blaNDM-5 gene via an IncX3-type plasmid among non-clonal Escherichia coli in China. Antimicrob. Resist. Infect. Control 7:59. doi: 10.1186/s13756-0180349-6

Liu, Z., Li, J., Wang, X., Liu, D., Ke, Y., Wang, Y., et al. (2018). Novel variant of new delhi metallo-beta-lactamase, NDM-20, in Escherichia coli. Front. Microbiol. 9:248. doi: 10.3389/fmicb.2018.00248

Livorsi, D. J., Chorazy, M. L., Schweizer, M. L., Balkenende, E. C., Blevins, A. E., Nair, R., et al. (2018). A systematic review of the epidemiology of carbapenemresistant Enterobacteriaceae in the United States. Antimicrob. Resist. Infect. Control 7:55. doi: 10.1186/s13756-018-0346-9

Long, H., Feng, Y., Ma, K., Liu, L., McNally, A., and Zong, Z. (2019). The co-transfer of plasmid-borne colistin-resistant genes mcr-1 and $\mathrm{mcr}-3.5$, the carbapenemase gene blaNDM-5 and the $16 \mathrm{~S}$ methylase gene rmtB from Escherichia coli. Sci. Rep. 9:696. doi: 10.1038/s41598-018-37125-1

Netikul, T., Sidjabat, H. E., Paterson, D. L., Kamolvit, W., Tantisiriwat, W., Steen, J. A., et al. (2014). Characterization of an IncN2-type blaNDM-(1)-carrying plasmid in Escherichia coli ST131 and Klebsiella pneumoniae ST11 and ST15 isolates in Thailand. J. Antimicrob. Chemother. 69, 3161-3163. doi: 10.1093/jac/ $\mathrm{dku} 275$

Otter, J. A., Burgess, P., Davies, F., Mookerjee, S., Singleton, J., Gilchrist, M., et al. (2017). Counting the cost of an outbreak of carbapenemase-producing Enterobacteriaceae: an economic evaluation from a hospital perspective. Clin. Microbiol. Infect. 23, 188-196. doi: 10.1016/j.cmi.2016.10.005

Peirano, G., Ahmed-Bentley, J., Fuller, J., Rubin, J. E., and Pitout, J. D. (2014). Travel-related carbapenemase-producing gram-negative bacteria in Alberta, Canada: the first 3 years. J. Clin. Microbiol. 52, 1575-1581. doi: 10.1128/JCM. 00162-14

Peirano, G., Mulvey, G. L., Armstrong, G. D., and Pitout, J. D. (2013). Virulence potential and adherence properties of Escherichia coli that produce CTX-M and NDM beta-lactamases. J. Med. Microbiol. 62, 525-530. doi: 10.1099/jmm. 0.048983-0

Poirel, L., Bonnin, R. A., and Nordmann, P. (2011). Analysis of the resistome of a multidrug-resistant NDM-1-producing Escherichia coli strain by highthroughput genome sequencing. Antimicrob. Agents Chemother. 55, 4224-4229. doi: 10.1128/AAC.00165-11

Qamar, M. U., Walsh, T. R., Toleman, M. A., Saleem, S., and Jahan, S. (2018). First identification of clinical isolate of a Novel "NDM-4" producing Escherichia coli ST405 from urine sample in Pakistan. Braz. J. Microbiol. 49, 949-950. doi: 10.1016/j.bjm.2018.02.009

Ranjan, A., Shaik, S., Mondal, A., Nandanwar, N., Hussain, A., Semmler, T., et al. (2016). Molecular epidemiology and genome dynamics of New Delhi metallobeta-lactamase-producing extraintestinal pathogenic Escherichia coli strains from India. Antimicrob. Agents Chemother. 60, 6795-6805. doi: 10.1128/AAC. 01345-16

Sun, F., Yin, Z., Feng, J., Qiu, Y., Zhang, D., Luo, W., et al. (2015). Production of plasmid-encoding NDM-1 in clinical Raoultella ornithinolytica and leclercia adecarboxylata from China. Front. Microbiol. 6:458. doi: 10.3389/fmicb.2015. 00458

Toleman, M. A., Bugert, J. J., and Nizam, S. A. (2015). Extensively drug-resistant New Delhi metallo-beta-lactamase-encoding bacteria in the environment, Dhaka, Bangladesh, 2012. Emerg. Infect. Dis. 21, 1027-1030. doi: 10.3201/ eid2106.141578

Wang, L. H., Liu, P. P., Wei, D. D., Liu, Y., Wan, L. G., Xiang, T. X., et al. (2016). Clinical isolates of uropathogenic Escherichia coli ST131 producing NDM-7 metallo-beta-lactamase in China. Int. J. Antimicrob. Agents 48, 41-45. doi: 10.1016/j.ijantimicag.2016.03.009

Wang, S., Zhao, S. Y., Xiao, S. Z., Gu, F. F., Liu, Q. Z., Tang, J., et al. (2016). Antimicrobial resistance and molecular epidemiology of Escherichia coli causing bloodstream infections in three hospitals in Shanghai, China. PLoS One 11:e0147740. doi: 10.1371/journal.pone.0147740

Wang, Q., Wang, X., Wang, J., Ouyang, P., Jin, C., Wang, R., et al. (2018). Phenotypic and genotypic characterization of carbapenem-resistant Enterobacteriaceae: data from a longitudinal large-scale CRE study in China (2012-2016). Clin. Infect. Dis. 67, S196-S205. doi: 10.1093/cid/ciy660

Wirth, T., Falush, D., Lan, R., Colles, F., Mensa, P., Wieler, L. H., et al. (2006). Sex and virulence in Escherichia coli: an evolutionary perspective. Mol. Microbiol. 60, 1136-1151. doi: 10.1111/j.1365-2958.2006.05172.x

Zhang, R., Liu, L., Zhou, H., Chan, E. W., Li, J., Fang, Y., et al. (2017). Nationwide surveillance of clinical carbapenem-resistant Enterobacteriaceae (CRE) strains in China. EBioMedicine 19, 98-106. doi: 10.1016/j.ebiom.2017.04.032

Zhang, X., Feng, Y., Zhou, W., McNally, A., and Zong, Z. (2018). Cryptic transmission of ST405 Escherichia coli carrying bla NDM-4 in hospital. Sci. Rep. 8:390. doi: 10.1038/s41598-017-18910-w

Zhong, L.-L., Stoesser, N., Doi, Y., Shen, C., Huang, X., and Tian, G.-B. (2017). Carriage of $\beta$-lactamase-producing Enterobacteriaceae by Chinese travellers. Lancet Infect. Dis. 17, 138-139. doi: 10.1016/S1473-3099(17) 30002-6

Zhu, Y. Q., Zhao, J. Y., Xu, C., Zhao, H., Jia, N., and Li, Y. N. (2016). Identification of an NDM-5-producing Escherichia coli sequence type 167 in a neonatal patient in China. Sci. Rep. 6:29934. doi: 10.1038/srep29934

Conflict of Interest Statement: The authors declare that the research was conducted in the absence of any commercial or financial relationships that could be construed as a potential conflict of interest.

Copyright (C) 2019 Hao, Shao, Geng, Bai, Jin and Lu. This is an open-access article distributed under the terms of the Creative Commons Attribution License (CC BY). The use, distribution or reproduction in other forums is permitted, provided the original author(s) and the copyright owner(s) are credited and that the original publication in this journal is cited, in accordance with accepted academic practice. No use, distribution or reproduction is permitted which does not comply with these terms. 